Irish Math. Soc. Bulletin

Number 84, Winter 2019, 19-20

ISSN 0791-5578

\title{
Proof-without-words: Markov's inequality
}

ROBIN E.HARTE, JANE HORGAN AND JAMES POWER

ABSTRaCT. We offer a "proof without words" of the inequalities of Markov and Chebyshev

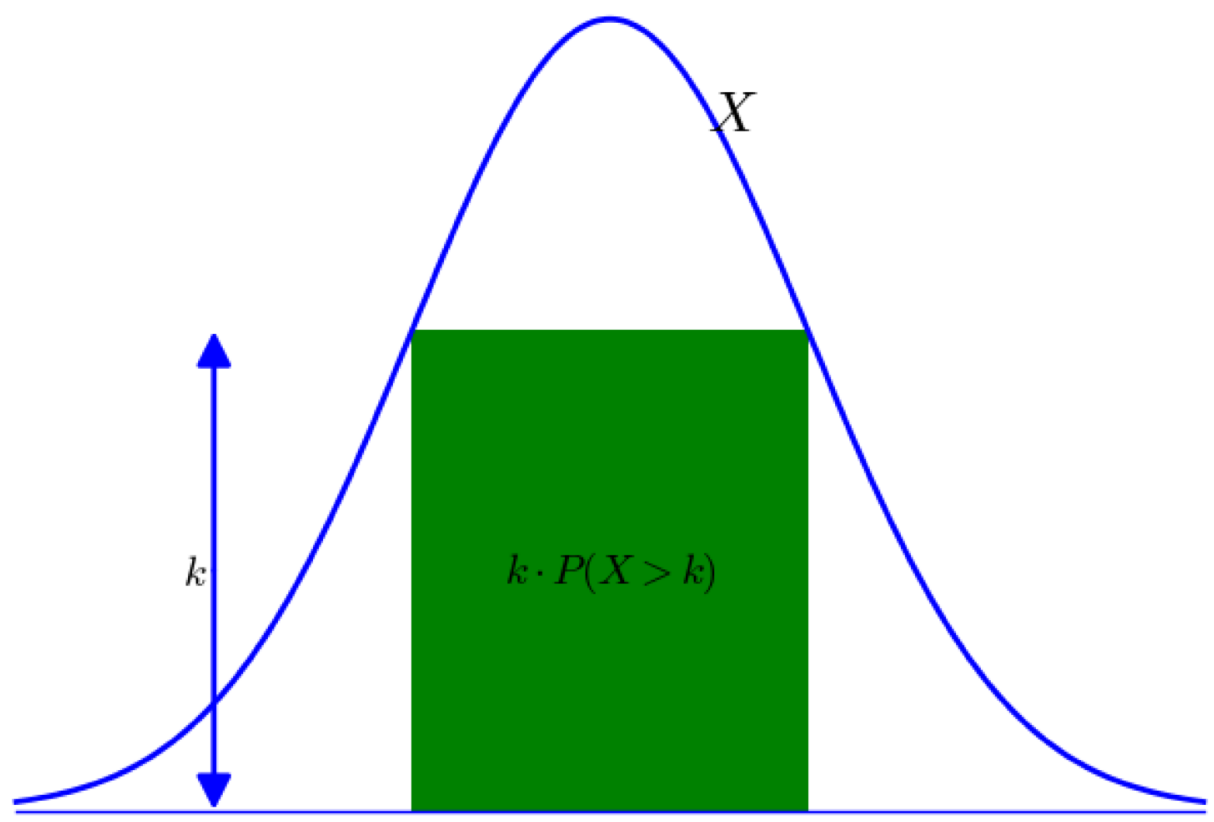

FiguRE 1. Markov's Inequality

Markov's inequality ([1] $\S 20.1$ ), for a random variable $X: \Omega \rightarrow[0, \infty) \subseteq \mathbb{R}$, living on a sample space $\Omega$ carrying a probability measure $P$, says that, if $0 \leq k \in \mathbb{R}$,

$$
k P(X \geq k) \leq E(X) .
$$

Here $E(X)$ is the expectation of $X$. The proof of (1) is rather simple: if

$$
F_{X}: t \mapsto P(X \leq t)
$$

is the cumulative distribution of $X$, then

$$
k P(X \geq k)=\int_{t=k}^{\infty} k d F_{X}(t) \leq \int_{t=k}^{\infty} t d F_{X}(t) \leq \int_{t=0}^{\infty} t d F_{X}(t)=E(X) .
$$

In our picture, necessarily set in two dimensional real paper, the total "area" under the graph of $X$ is the expectation $E(X)$, while the product $k(P(X \geq k)$ is the area of the

2010 Mathematics Subject Classification. 60F05; 28A10, $28 \mathrm{C} 05$.

Key words and phrases. Markov inequality, Chebyshev inequality, sample space, cumulative distribution.

Received on 20-11-2019; revised 13-12-20. 
shaded "rectangle". We do not assume that $\Omega \subseteq \mathbb{R}$, and certainly not that the base of the rectangle is a single real interval.

A proof of Markov's inequality is also a proof of Chebyshev's inequality: Chebyshev's inequality for $X$ is neither more nor less than Markov's inequality for $\varphi(X)$, where, if $0 \leq t \in \mathbb{R}$

$$
\varphi(t)=|t-E(X)|^{2} .
$$

In the middle of this writing the third author suddenly left us, far, far too soon: we offer it now as a tribute.

\section{REFERENCES}

[1] Jane M. Horgan, Probability with R, Wiley, 2009

Robin E. Harte taught for twenty years at University College, Cork, and retired a long time ago, but somehow never goes away; his ghost can sometimes be seen at the TCD-UCD Analysis Seminar.

Jane Horgan was in at the beginning of NIHE Dublin, now DCU, where she helped to set up the School of Computer Applications, and initiated the Ballymun Access Scheme for admissions to Dublin City University. In some sort of retirement, she has developed an obsession with the Turkish language, and the second edition of Probability with $R$ is about to hit the shelves.

James Power taught Computer Science in DCU for a number of years before moving to Maynooth University in 2005. His research centred on parsers and front-end compiler technology for object-oriented languages, and also fuzzy systems. Computer Science in Ireland, and the academic world generally, are diminished by his sudden and untimely death, of a heart attack, in late August 2019.

(R. Harte) School of Mathematics, TCD.

(J. Horgan) School of Computing, Dublin City University, Dublin 9

E-mail address, R. Harte: rharte@maths.tcd.ie

E-mail address, J. Horgan: janemhorgan@gmail.com 